\title{
Numerical method for real root isolation of semi-algebraic system and its applications th
}

\author{
Zhenyi $\mathrm{Ji}^{1,2 *}$, Wenyuan $\mathrm{Wu}^{2}$, $\mathrm{Yi} \mathrm{Li}^{2}$, Yong Feng ${ }^{2}$ \\ ${ }^{1}$ Lab. of Computer Reasoning and Trustworthy Comput, School of Computer Science and \\ Engineering, University of Electronic Science and Technology of China, Chengdu 611731, \\ P.R.China \\ ${ }^{2}$ Lab. of Automated Reasoning and Cognition, Chongqing Institute of Green and \\ Intelligent Technology, Chinese Academy of Science, Chongqing, 401120, P.R. China
}

\begin{abstract}
In this paper, based on the homotopy continuation method and the interval Newton method, an efficient algorithm is introduced to isolate the real roots of semi-algebraic system. Tests on some random examples and a variety of problems including transcendental functions arising in many applications show that the new algorithm reduces the cost substantially compared with the traditional symbolic approaches.
\end{abstract}

Keywords:

Semi-algebraic system; Homotopy method; Interval Newton's method; Real root isolation

\section{Introduction}

The problem of counting and isolating real solutions of nonlinear system is an important topic in computing geometry and many other applications in various fields, e.g., the real intersection points for piecewise algebraic curve [1, 2, 3, 4, 5], the stability of a large class of biological networks [6, 7],

\footnotetext{
«This research was partially supported by the National Natural Science Foundation of China(11171053), and the National Natural Science Foundation of China Youth Fund Project(11001040,61103110)

${ }^{*}$ Corresponding author

Email address: zyji001@163.com (Zhenyi $\mathrm{Ji}^{1,2}$ )
} 
discovering non-linear ranking functions of loop programs in [ 8 , 9], automated proving inequality type theorem [10] and so on.

Many algorithms for real root isolation of one polynomial in one variable have been developed in [11, 12, 13, 14, 15]. For multivariable case, Xia et al.[16] proposed an algorithm based on Wu's method for isolating the real roots of semi-algebraic system with integer coefficients, and made it more available with interval algorithm in their later work [17]. There are also other algorithms based on different techniques, see [18, 19, 20, 21] for more details.

Actually, most of the algorithms mentioned above can compute the exact results because they depend on symbolic computations, but they are restricted to small size systems because of the high complexity of the symbolic computation. In order to avoid this problem, Shen et al. 22] presented a numerical algorithm improving the efficience based on homotopy continuation method combined with interval Newton iteration technique.

In this paper, we extend the numerical method to a class of semi-algebraic systems and transcendental functions. We always assume that the system is square and it only has isolated roots in $\mathbb{C}$. In order to avoid the singularity of the Jacobian matrix, we also suppose that the multiplicity of these points are one.

The rest of the paper is organized as follows. Definitions and preliminaries about homotopy continuation method and interval arithmetic are given in section 2. Section 3 first gives a brief review of the numerical algorithm for isolating real roots of a class of polynomial systems, and then presents the numerical method for isolating the real roots of semi-algebraic systems. The experimental results together with comparison to symbolic methos is given in this section. Applying the new algorithm to some problems arising from piecewise algebraic curve, chemical engineering, robot kinematic problem, circuit design are shown in section 4 . Finally, section 5 draws a conclusion of this paper.

\section{Interval Arithmetic And Homotopy Continuation Method}

In this section, some basic theories and tools about interval arithmetic and homotopy continuation method are presented. 


\subsection{Interval Newton's method}

Interval operations were first introduced by Moore [23]. The key idea of interval arithmetic is substituting an interval for a floating number in numerical computation, which is used to tackle the instability and error analysis. For a more detailed and complete discussion the reader is referred to [24].

A real interval $X$ is a nonempty set of real numbers

$$
X=[\underline{x}, \bar{x}]=\{x \in \mathbb{R}: \underline{x}<x<\bar{x}\},
$$

the set of all intervals in $\mathbb{R}$ is denoted by $I(\mathbb{R})$.

An interval vector $\mathbf{X}$ is a vector whose elements are intervals, and an interval matrix can be similarly defined. In the rest of this paper, we let $\mathrm{X}$ denotes the set of interval vector.

For an interval $X$, the midpoint of $X$ is $m(X)=(\underline{x}+\bar{x}) / 2$, the width of $X$ is $w(x)=\bar{x}-\underline{x}$, and the radius of $X$ is $r(X)=(\bar{x}-\underline{x}) / 2$.

Given interval $X=[\underline{x}, \bar{x}], Y=[\underline{y}, \bar{y}]$, the four element operations are defined as

$$
\begin{aligned}
& X+Y=[\underline{x}+\underline{y}, \bar{x}+\bar{y}] \\
& X-Y=[\underline{x}-\bar{y}, \bar{x}-\underline{y}] \\
& X \cdot Y=[\min \{\underline{x} \underline{y}, \underline{x} \bar{y}, \bar{x} \underline{y}, \bar{x} \bar{y}\}, \max \{\underline{x} \underline{y}, \underline{x} \bar{y}, \bar{x} \underline{y}, \bar{x} \bar{y}\}] \\
& X / Y=[\underline{x}, \bar{x}] \cdot[1 / \underline{y}, 1 / \bar{y}], 0 \notin[\underline{y}, \bar{y}] .
\end{aligned}
$$

The intersection of two intervals $X$ and $Y$ is empty if either $\bar{x}<\underline{y}$ or $\bar{y}<\underline{x}$. In this case, we write $X \cap Y=\emptyset$.

For interval matrices and interval vectors, the concepts such as midpoint, width, radius, etc, and the arithmetic operations are defined in components.

Let $\mathbb{R}[\boldsymbol{x}]$ be the ring of polynomials in the variables $\left(x_{1}, x_{2}, \cdots, x_{n}\right)$ with coefficients in $\mathbb{R}$, and $\boldsymbol{f}=\left[f_{1}, \cdots, f_{n}\right]$ be a polynomial system, where $f_{i} \in$ $\mathbb{R}[\boldsymbol{x}]$.

Suppose $f: \mathbb{R}^{n} \rightarrow \mathbb{R}$ is a function from a real vector to a real number, $F: I\left(\mathbb{R}^{n}\right) \rightarrow I(\mathbb{R})$ is called an interval extension of $f$ if

$$
F\left(\left[x_{1}, x_{1}\right], \cdots,\left[x_{n}, x_{n}\right]\right)=f\left(x_{1}, \cdots, x_{n}\right)
$$

for all $x_{i} \in X_{i}, i=1,2, \ldots, n$.

We say that $F=F\left(X_{1}, \cdots, X_{n}\right)$ is inclusion monotonicity if

$$
Y_{i} \subseteq X_{i}, i=1, \cdots, n \Rightarrow F\left(Y_{1}, \cdots, Y_{n}\right) \subseteq F\left(X_{1}, \cdots, X_{n}\right) .
$$


And it is easy to prove that all the polynomial operations satisfy the inclusive monotonicity.

In the following, we let $\boldsymbol{f}^{\prime}$ be the Jacobian matrix of $\boldsymbol{f}$, and $\boldsymbol{F}, \boldsymbol{F}^{\prime}$ be the interval extension of $\boldsymbol{f}$ and $\boldsymbol{f}^{\prime}$ with inclusive monotonicity, respectively.

Moore first define the following interval Newton's operators:

$$
N(\mathbf{X})=m(\mathbf{X})-V(\mathbf{X}) \boldsymbol{f}(m(\mathbf{X}))
$$

where $V(\mathbf{X})$ is an interval matrix containing $\boldsymbol{F}^{\prime}(\mathbf{X})^{-1}$.

In order to avoid the computation of the inversion of interval matrix in formula (1), Krawcayk proposed the following operator:

$$
K(\boldsymbol{y}, \mathbf{X})=\boldsymbol{y}-\mathrm{Y} \boldsymbol{f}(\boldsymbol{y})+\left(\mathbf{I}-\mathrm{Y} \boldsymbol{F}^{\prime}(\mathbf{X})\right)(\mathbf{X}-\boldsymbol{y}),
$$

where $\boldsymbol{y}$ is chosen from the region $\mathbf{X}, \mathbf{I}$ denotes the unit matrix, and $\mathrm{Y}$ is an arbitrary nonsingular matrix.

It has been proved that Krawcayk operator has the following properties.

Proposition 2.1. Suppose $K(\boldsymbol{y}, \boldsymbol{X})$ is computed by formula (2), then

1): $\boldsymbol{x}^{*} \in \boldsymbol{X}$ and $\boldsymbol{f}\left(\boldsymbol{x}^{*}\right)=0 \Rightarrow \boldsymbol{x}^{*} \in K(\boldsymbol{y}, \boldsymbol{X})$.

2): $K(\boldsymbol{y}, \boldsymbol{X}) \subset \operatorname{int}(\boldsymbol{X}) \Rightarrow \boldsymbol{f}$ has only one root in $\boldsymbol{X}$, where int $(\boldsymbol{X})$ denotes the topological interior of the box of $\boldsymbol{X}$.

3): $K(\boldsymbol{y}, \boldsymbol{X}) \subseteq \operatorname{int}(\boldsymbol{X}) \Rightarrow \boldsymbol{f}$ has a root in $\boldsymbol{X}$.

4): $K(\boldsymbol{y}, \boldsymbol{X}) \cap \boldsymbol{X}=\varnothing \Rightarrow$ no solution in $\boldsymbol{X}$.

In particular, if $\boldsymbol{y}$ and $\mathbf{Y}$ are chosen to be $\mathbf{y}=m(\mathbf{X})$ and $\mathbf{Y}=\left[m\left(\boldsymbol{F}^{\prime}(\mathbf{X})\right)\right]^{-1}$ respectively, then the Moore form of the Krawcayk operators is :

$$
K(\mathbf{X})=m(\mathbf{X})-\left[m\left(\boldsymbol{F}^{\prime}(\mathbf{X})\right)\right]^{-1} \boldsymbol{f}(m(\mathbf{X}))+\Delta,
$$

where $\Delta=\left(\mathbf{I}-\left[m\left(\boldsymbol{F}^{\prime}(\mathbf{X})\right)\right]^{-1} \boldsymbol{F}^{\prime}(\mathbf{X})\right)(\mathbf{X}-m(\mathbf{X}))$.

\subsection{Homotopy continuation method}

Homotopy continuation method is an efficient numerical method for finding all isolated solutions of polynomial system. The method traces a path from the solution of an easy problem to the solution of the given problem by use of a homotopy continuous transformation. See reference [26, 27, 28, 29, 30] for more details. There also exists some software packages [31, 32, 33] for homotopy continuation methods. In our implementation, we use Hom4ps-2.0 which could return all the approximate complex zeros of a given polynomial system efficiently, along with residues and condition numbers. 


\section{Algorithm for isolating real zeros of semi-algebraic system}

In this section, we will present an algorithm for isolating the real roots of a zero-dimensional semi-algebraic system. Our idea is to compute the isolated real root intervals of the zero-dimensional polynomial system first. In this step, we will use the hybrid algorithm introduced by Shen et, al [22]. Then decide whether these intervals satisfy inequations by substitution.

First, we introduce the definition of semi-algebraic system. A semialgebra system denoted by $S A S$ can be written in the following form:

$$
\left\{\begin{array}{l}
f_{1}=0, \cdots, f_{n}=0, \\
p_{1}>0, \cdots, p_{q}>0, \\
n_{1} \geq 0, \cdots, n_{s} \geq 0, \\
h_{1} \neq 0, \cdots, h_{t} \neq 0,
\end{array}\right.
$$

where $n \geq 1$ and $q, s, t \geq 0$. We call it zero-dimensional semi-algebraic system if $\left\{f_{1}, \cdots, f_{n}\right\}$ has only finite zeros in $\mathbb{C}$. Following the notations in software package Discover [16], we let $\boldsymbol{f}, \boldsymbol{n}, \boldsymbol{p}, \boldsymbol{h}$ denote the polynomial equations, non-negative polynomial inequalities, positive polynomial inequalities and polynomial inequations respectively.

\subsection{Real root isolation for zero-dimensional polynomial system}

In this subsection, we introduce a hybrid method for the real root isolation of a zero-dimensional polynomial system, see [22] for more details.

Suppose we have obtained all the isolated approximate roots of $\boldsymbol{f}$ through homotopy continuation method. For each approximate root, the following proposition gives a method to construct initial interval which contains its corresponding accurate root.

Proposition 3.1. [22] Let $\boldsymbol{f}=\left[f_{1}, \cdots, f_{n}\right]$ be a polynomial system in $\mathbb{R}[\boldsymbol{x}]$, and $\overline{\boldsymbol{x}} \in \mathbb{R}^{n}$ be an approximate zero of $\boldsymbol{f}$. If the following conditions hold:

1: $\boldsymbol{f}^{\prime}(\overline{\boldsymbol{x}})$ exists, and there are real numbers $B$ and $\eta$ such that

$$
\left\|\boldsymbol{f}^{\prime}(\overline{\boldsymbol{x}})^{-1}\right\| \leq B,\left\|\boldsymbol{f}^{\prime}(\overline{\boldsymbol{x}})^{-1} \boldsymbol{f}(\overline{\boldsymbol{x}})\right\| \leq \eta,
$$

2: There exists a ball neighbourhood $O(\overline{\boldsymbol{x}}, \omega)$ such that $\boldsymbol{f}^{\prime}(\boldsymbol{x})$ satisfies the Lipschitz condition on it:

$$
\left\|\boldsymbol{f}^{\prime}(\boldsymbol{x})-\boldsymbol{f}^{\prime}(\boldsymbol{y})\right\| \leq K\|\boldsymbol{x}-\boldsymbol{y}\|, \forall \boldsymbol{x}, \boldsymbol{y} \in O(\overline{\boldsymbol{x}}, \omega)
$$


3:

$$
h=B K \eta \leq \frac{1}{2}, \omega \geq \frac{1-2 \sqrt{1-2 h}}{h} \eta,
$$

then $\boldsymbol{f}$ has only one root in $\overline{O(\overline{\boldsymbol{x}}, \omega)}$.

Let $\hat{\boldsymbol{x}}$ be an accurate root, and $\overline{\boldsymbol{x}}$ be its approximation. Denote the Hessian matrix of $f_{j}$ by $H_{j}$, and $h_{j}^{i}$ its column vector. Let

$$
\lambda=\max _{1 \leq i \leq n} \sum_{j=1}^{n}\left|h_{j}^{i}(\overline{\boldsymbol{x}})\right|_{\text {max }}, \text { and } r=\frac{\left.\left\|J_{F}^{-1}(\overline{\boldsymbol{x}})\right\|\right|_{\infty}\left\|J_{F}(\overline{\boldsymbol{x}})\right\|_{\infty}^{2}}{1-n \lambda\left\|J_{F}^{-1}(\overline{\boldsymbol{x}})\right\|_{\infty}\left\|J_{F}(\overline{\boldsymbol{x}})\right\|_{\infty}}
$$

where $|\cdot|_{\text {max }}$ denotes the maximum module component of a vector.

A natural fact about $r$ is that if $r>\operatorname{Im}(\overline{\boldsymbol{x}})$, then $\overline{\boldsymbol{x}}$ is a imaginary number, where $\operatorname{Im}(\overline{\boldsymbol{x}})$ means the imaginary part of $\overline{\boldsymbol{x}}$. So we can delete some complex roots without using the interval arithmetic.

We use algorithms from [22] to compute $\omega$ and $\lambda$.

According to the above descriptions, the following algorithm real_roots can be used for isolating real root intervals of $f$.

Algorithm 1 : real_root_isolate [22]

Input: Polynomial system: $\boldsymbol{f}$, and a threshold $\tau$.

Output: Isolated intervals of $\boldsymbol{f}$ real_roots or \{\} .

1. Let real_roots $=\{\}$.

2. Computing all the isolated roots of $\boldsymbol{f}$, denote it by roots, and let nreal be the number of roots.

3. If $n r e a l=0$, then return real_roots, and stop this program.

4. For $i=1$ : nreal;

- let $z$ be the $i$ th element of roots, and determine whether $z$ is a complex root using formula (5) and $\operatorname{Im}(z)$.

- If $z$ is a complex root, stop; else, construct the initial interval of the real part of $z$ according to proposition 3.1, and denote it by $\mathbf{X}_{0}$. Compute $K\left(\mathbf{X}_{0}\right)$ according to formula (3). If $K\left(\mathbf{X}_{0}\right) \subset \mathbf{X}_{0}$, then let real_roots $=$ real_roots $\cup\left\{K\left(\mathbf{X}_{0}\right) \cap \mathbf{X}_{0}\right\}$, and stop this step. If $K\left(\mathbf{X}_{0}\right) \cap \mathbf{X}_{0}=\varnothing$, stop. Otherwise, bisect $\mathbf{X}_{0}=K(\mathbf{X}) \cap \mathbf{X}_{0}$, and process each half separately.

5. End For 
6. If there exists some intervals in real_root such that they are not disjoint, then compute the intersection of these intervals, split each interval $\mathbf{X}_{i}=\mathbf{X}_{i 1} \cup \mathbf{X}_{i 2}$, where $\mathbf{X}_{i 1}$ denotes the intersection portion, process all the subintervals, and remove the subinterval which don't contain a real root of $\boldsymbol{f}$.

7. If the width of an interval is bigger than $\tau$, then bisect the interval and process each half separately.

8. Return real_roots.

\subsection{Algorithm for semi-algebraic system}

Now suppose we have obtained the isolated real root intervals of polynomial system $\boldsymbol{f}$ defined in (4). Let $\mathbf{X}=\left(\left[a_{1}, b_{1}\right], \cdots,\left[a_{n}, b_{n}\right]\right)$ be an isolated real root interval of $\boldsymbol{f}$, and $\hat{\boldsymbol{x}}$ be the accurate zero of $\boldsymbol{f}$ such that $\hat{\boldsymbol{x}} \in \mathbf{X}$. Assume $f$ is a positive polynomial inequality in (4) and $f(\mathbf{X})=[a, b]$, then there are only three cases that can happen about the sign of $[a, b]$, including the following:

1: $a>0 \Rightarrow f(\hat{\boldsymbol{x}})>0$.

2: $b<0 \Rightarrow f(\hat{\boldsymbol{x}})<0$.

3: $0 \in[a, b]$.

Hence, if $[a, b]$ satisfies the first case or the second, we can easily decide whether $\mathbf{X}$ satisfy $f$ or not. For case 3 , it is difficult to decide the sign of $f(\hat{\boldsymbol{x}})$. In the following, we present a method to solve this problem.

Let $g=y^{2} f+1$ be a new polynomial in $\mathbb{R}[\boldsymbol{x}, y]$, and $[\boldsymbol{f}, g]$ be a polynomial system $\left\{f_{1}, \cdots, f_{n}, g\right\}$ in $\mathbb{R}[\boldsymbol{x}, y]$, where $\mathbb{R}[\boldsymbol{x}, y]$ denotes the ring of polynomials in the variables $\left(x_{1}, x_{2}, \cdots, x_{n}, y\right)$ with coefficients in $\mathbb{R}$. Assume $\mathbf{Z}=\left\{\mathbf{Z}_{1}, \cdots, \mathbf{Z}_{m}\right\}$ is the set of the isolated real root intervals of $[\boldsymbol{f}, g]$. Define the projection $\pi: I\left(\mathbb{R}^{n+1}\right) \rightarrow I\left(\mathbb{R}^{n}\right)$ which remove the last coordinate of an interval vector in $I\left(\mathbb{R}^{n+1}\right)$.

Theorem 3.1. If the intersection of $\boldsymbol{X}$ and $\pi\left(\boldsymbol{Z}_{i}\right)$ is an empty set for all $i \in\{1,2, \cdots, m\}$, then $f(\hat{\boldsymbol{x}}) \geq 0$.

Proof. Denote $\boldsymbol{X}$ by $\left(\left[a_{1}, b_{1}\right], \cdots,\left[a_{n}, b_{n}\right]\right)$ and $\boldsymbol{Z}_{i}=\left(\left[a_{i 1}, b_{i 1}\right], \cdots,\left[a_{i n}, b_{i n}\right]\right.$, $\left.\left[y_{i 1}, y_{i 2}\right]\right), i=1,2, \cdots, m$.

Suppose $f(\hat{\boldsymbol{x}})<0$, then it is easy to see that the point $(\hat{\boldsymbol{x}}, \sqrt{-1 / f(\hat{\boldsymbol{x}})})$ is the accurate zero of $[\boldsymbol{f}, g]$, so there exists an isolated real root interval $\boldsymbol{Z}_{i}$ which contains the point $(\hat{\boldsymbol{x}}, \sqrt{-1 / f(\hat{\boldsymbol{x}})})$. Hence the intersection of $\boldsymbol{X}$ and $\pi\left(\boldsymbol{Z}_{i}\right)$ is not an empty set, which contradicts to our suppose, so $f(\hat{\boldsymbol{x}}) \geq 0$. This proves the first part of the theorem. 
On the other hand, assume $f(\hat{\boldsymbol{x}}) \geq 0$, then $g=y^{2} f(\hat{\boldsymbol{x}})+1 \geq 1$ for any real value of $y$, so $\pi\left(\boldsymbol{Z}_{i}\right)$ does't contain $\hat{\boldsymbol{x}}$ for all $i \in\{1, \cdots, m\}$. This establishes that the intersection of $\mathbf{X}$ and $\pi\left(\boldsymbol{Z}_{i}\right)(i=1, \cdots, m)$ is an empty set. This completes the proof.

Similar to theorem 3.1, if we construct $g=y^{2} f-1$, then we can decide whether $f(\hat{\boldsymbol{x}}) \leq 0$ or not.

According to the above theorem, the following Deter_Sign can be used to determine the sign of $f(\hat{\boldsymbol{x}})$ if $0 \in[a, b]$.

Algorithm 2: Deter_Sign

Input: A polynomial system $\boldsymbol{f}$; An isolated real root interval $\boldsymbol{X}$ of $\boldsymbol{f}$; A polynomial $f$, and an index $I$, the value of $I$ is 1 or -1 .

Output: If $f(\hat{\boldsymbol{x}}) I \geq 0$, then return 1 ; else return -1 ;

1. If $\mathrm{I}=1$

- Let $g=y^{2} f+1$;

2. Else

- Let $g=y^{2} f-1$;

3. End If

4. real_roots $=$ real_root_isolate $([\boldsymbol{f}, g])$;

5. $\operatorname{sum}=0$;

6. For $i=1$ : length(real_roots)

- If $\boldsymbol{X} \cap \pi($ real_roots $\{i\})=\emptyset$, then sum $=$ sum +1 ;

- Else, return -1;

- End If

7. End For

8. If sum $=$ nreal then return 1 ;

9. End If

Using algorithm 2, it is easy to see that if

$$
\operatorname{Deter\_ sign}(\boldsymbol{f}, f, \boldsymbol{X}, 1)=1 \wedge \text { Deter_sign }(\boldsymbol{f}, f, \boldsymbol{X},-1)=1,
$$

then $f(\hat{\boldsymbol{x}})=0$.

In the following, a simple example is given to explain how the algorithm determines the sign of $f(\hat{\boldsymbol{x}})$. 
Example 3.1. Let $\boldsymbol{f}=\left[x^{2}+y-2, x+2 y-3\right]$, and $h=3 x+y-4$. It is easy to see that $\boldsymbol{f}$ and $h$ has a common point $(1,1)$.

Using algorithm 1, we obtain an isolated real root interval of $\boldsymbol{f}$ which contains the point $(1,1)$.

$$
\begin{aligned}
\boldsymbol{X}_{1}= & {[0.999999999999900,1.000000000000100], } \\
& {[0.999999999999900,1.000000000000100] . }
\end{aligned}
$$

Substituting $\boldsymbol{X}_{1}$ into $h$, we have

$$
h\left(\boldsymbol{X}_{1}\right)=10^{-11} \times[-0.400124378074906,0.399680288865056] .
$$

Now, let $h_{1}=a^{2}(3 x+y-4)+1$, then the isolated real root intervals of $\left[\boldsymbol{f}, h_{1}\right]$ are

$$
\begin{aligned}
\boldsymbol{Z}_{1}= & {[-0.500000000000100,-0.499999999999900], } \\
& {[1.749999999999900,1.750000000000100], } \\
& {[-0.516397779494422,-0.516397779494222] . } \\
\boldsymbol{Z}_{2}= & {[-0.500000000000100,-0.499999999999900], } \\
& {[1.749999999999900,1.750000000000100], } \\
& {[0.516397779494422,0.516397779494222] . }
\end{aligned}
$$

So $\boldsymbol{X}_{1} \cap \pi\left(\boldsymbol{Z}_{1}\right)=\varnothing$ and $\boldsymbol{X}_{2} \cap \pi\left(\boldsymbol{Z}_{2}\right)=\varnothing$, then we have $h(1,1) \geq 0$. Similarly, we obtain $h(1,1) \leq 0$ through isolating the real roots of $\left[\boldsymbol{f}, a^{2}(3 x+\right.$ $y-4)-1$. Hence $h(1,1)=0$.

Now, we give the following algorithm for computing the isolated intervals which satisfy the inequations $\boldsymbol{h}$ in (4).

Algorithm 3: Dele_Inequ

Input: Polynomial system $\boldsymbol{f}$ and $\boldsymbol{h}$; isolated real root intervals real_roots of $f$.

Output: real_roots $=\{\boldsymbol{X} \in$ real_roots $\mid \boldsymbol{h}(\hat{\boldsymbol{x}}) \neq 0, \boldsymbol{f}(\hat{\boldsymbol{x}})=0, \hat{\boldsymbol{x}} \in \boldsymbol{X}\}$.

1. Set Index $=\{\}$;

2. For $s=1$ : length(real_roots)

- Substituting real_roots $\{s\}$ into $\boldsymbol{h}$, and suppose $\boldsymbol{h}($ real_roots $\{s\})=\left(\left[a_{1}, b_{1}\right], \cdots,\left[a_{t}, b_{t}\right]\right)$;

- Let set $=\left\{i \in\{1, \cdots, t\} \mid 0 \in\left[a_{i}, b_{i}\right]\right\}$;

- If length $($ set $) \neq t$, then break; 
- End if

- For $t=1$ : length(set)

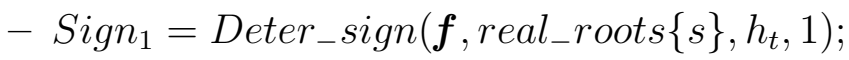

- If $\operatorname{sign}_{1}=1$, then

$\operatorname{Sign}_{2}=\operatorname{Deter}_{-} \operatorname{sign}\left(\boldsymbol{f}\right.$, real_roots $\left.\{s\}, h_{t},-1\right)$;

$*$ If $\operatorname{sign}_{2}=1$, then Index $=$ Index $\bigcup\{s\}$, break;

* End If

- End If

- End For

3. End For;

4. real_roots $=$ real_roots $\backslash$ real_roots $($ Index $)$.

In the following, we will present a method to deal with the non-negative systems $\boldsymbol{n}$ in semi-algebraic system defined in (4).

Algorithm 4 : Dele_Nonnega

Input: Two Polynomial systems: $\boldsymbol{f}$ and $\boldsymbol{n}$; isolated real root intervals of $f$ : real_roots .

Output: real_roots $=\{\boldsymbol{X} \in$ real_roots $\mid \boldsymbol{n}(\hat{\boldsymbol{x}}) \geq 0, \boldsymbol{f}(\hat{\boldsymbol{x}})=0, \hat{\boldsymbol{x}} \in \boldsymbol{X}\}$.

1. Set Index $=\{\}$;

2. For $t=1$ : length(real_roots)

- Substituting real_roots $\{t\}$ into $\boldsymbol{n}$, and suppose $\boldsymbol{n}($ real_roots $\{t\})=\left(\left[a_{1}, b_{1}\right], \cdots,\left[a_{s}, b_{s}\right]\right)$;

- If there exists some $i \in\{1, \cdots, s\}$ such that $b_{i}<0$, then let Index $=$ Index $\cup\{t\}$;

- Else if there exists some $i \in\{1, \cdots, s\}$ such that $0 \in\left[a_{i}, b_{i}\right]$. Let set $=\left\{i \in\{1, \cdots, s\} \mid 0 \in\left[a_{i}, b_{i}\right]\right\}$;

- For $j=1$ : length(set)

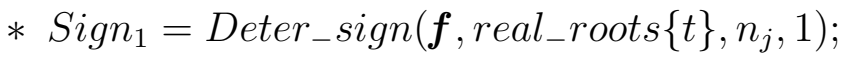

$*$ If $\operatorname{sign}_{1}=-1$, then Index $=$ Index $\cup\{s\}$, break;

* End If

- End for

- End if

3. End for 
4. $r e a l_{-}$roots $=$real_roots $\backslash$ real_roots $($ Index $)$.

The following algorithm is to remove the intervals which satisfy $\boldsymbol{p}$ in (4). Algorithm 5: Dele_Posi

Input: Two polynomial systems: $\boldsymbol{f}$ and $\boldsymbol{p}$; isolated intervals real_roots of $f$;

Output: real_roots $=\{\boldsymbol{X} \in$ real_roots $\mid \boldsymbol{p}(\hat{\boldsymbol{x}})>0, \boldsymbol{f}(\hat{\boldsymbol{x}})=0$, where $\hat{\boldsymbol{x}} \in$ $\boldsymbol{X}\}$.

1. real_roots $=$ Dele_Inequ $(\boldsymbol{f}$, real_roots, $\boldsymbol{p})$;

2. real_roots $=$ Dele_Nonnega $(\boldsymbol{f}$, real_roots, $\boldsymbol{p})$;

Until now, we have described all the parts of the algorithm for isolating the real roots of semi-algebraic system. Here, we present the whole algorithm in the following.

Algorithm 6: real_root_semi

Input: A semi-algebraic system in the form of (4).

Output: isolated real root intervals of (4) or return \{\} ;

1. real_roots $=$ real_root_isolate $(\boldsymbol{f})$;

2. If length $($ real_roots $)=0$, then return \{\} ;

3. Else

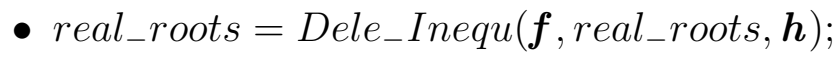

- If length(real_roots) $=0$, then return \{\} ;

- Else

- real_roots $=$ Dele_Nonnega $(\boldsymbol{f}$, real_roots, $\boldsymbol{n})$;

- If length(real_roots $)=0$, then return \{\} ;

- Else

* real_roots $=$ Dele_Posi $(\boldsymbol{f}$, real_roots, $\boldsymbol{p})$;

$*$ If length(real_roots $)=0$, then return \{\} ;

* End If

- End If

- End If

4. End If

5. Return real_roots. 
In the following, a simple example is provided to illustrate algorithm 6 for isolating the real roots of a semi-algebraic system.

Example 3.2. Given a semi-algebraic system,

$S A S=\left\{\begin{aligned} f_{1} & =5+13 x_{1}-10 x_{2}-82 x_{1}^{2}+71 x_{1} x_{2}+16 x_{2}^{2} . \\ f_{2} & =403.22 x_{1}-314.64+73.16 x_{1}^{2}-269.26 x_{1} x_{2}+300.96 x_{2}- \\ & 48 x_{1}^{3}+53 x_{1}^{2} x_{2}-28 x_{2}^{2} x_{1}+95.76 x_{2}^{2} . \\ & x_{1}-0.3>0, x_{2}>0, x_{1}-3.42 \neq 0 .\end{aligned}\right.$

Step 1: Denote the isolated real root intervals of $\left[f_{1}, f_{2}\right]$ by $\mathrm{X}$, using algorithm 1, we obtain six intervals:

$$
\begin{aligned}
\boldsymbol{X}_{1}= & {[3.419999999999901,3.420000000000101], } \\
& {[3.202328957744943,3.202328957745143] ; } \\
\boldsymbol{X}_{2}= & {[3.419999999999901,3.420000000000101], } \\
& {[-17.753578957745148,-17.753578957744949] ; } \\
\boldsymbol{X}_{3}= & {[-0.099812907756173,-0.099812907755973], } \\
& {[0.857641152899082,0.857641152899282] ; } \\
\boldsymbol{X}_{4}= & {[-8.128471753948865,-8.128471753948666], } \\
& {[-7.758843802156950,-7.758843802156750] ; } \\
\boldsymbol{X}_{5}= & {[0.584413218566582,0.584413218566782], } \\
& {[-2.373999555338525,-2.373999555338325] ; } \\
\boldsymbol{X}_{6}= & {[0.560588744512268,0.560588744512469], } \\
& {[0.376338245290841,0.376338245291041] . }
\end{aligned}
$$

Step 2: Substituting X into $x_{1}-3.42$, we have $e v_{1}=[-0.000000000000099,0.000000000000100]$, $e v_{2}=[-0.000000000000099,0.000000000000101]$, $e v_{3}=[-3.519812907756173,-3.519812907755973]$, $e v_{4}=[-11.548471753948863,-11.548471753948665]$, $e v_{5}=[-2.835586781433418,-2.835586781433218]$, $e v_{6}=[-2.859411255487732,-2.859411255487531]$.

It is easy to see that $x_{1}-3.42 \neq 0$ at any point contained in intervals $\boldsymbol{X}_{3}, \boldsymbol{X}_{4}, \boldsymbol{X}_{5}, \boldsymbol{X}_{6}$, so these intervals should be retained. Using algorithm 3 to process intervals $\boldsymbol{X}_{1}$ and $\boldsymbol{X}_{2}$, we know that $x_{1}-3.42$ is equal to zero at the solutions of $\left\{f_{1}, f_{2}\right\}$, so we discard $\boldsymbol{X}_{1}$ and $\boldsymbol{X}_{2}$ at this step. 
Step 3: Substituting $\boldsymbol{X}_{3} \boldsymbol{X}_{4} \boldsymbol{X}_{5}$ and $\boldsymbol{X}_{6}$ into $\boldsymbol{P}=\left\{x_{1}-0.3, x_{2}\right\}$, we obtain the following four intervals

$$
\begin{aligned}
\boldsymbol{P}\left(\boldsymbol{X}_{3}\right)= & {[-0.399812907756173,-0.399812907755973], } \\
& {[0.857641152899082,0.857641152899282] ; } \\
\boldsymbol{P}\left(\boldsymbol{X}_{4}\right)= & {[-8.428471753948864,-8.428471753948665], } \\
& {[-7.758843802156949,-7.758843802156748] ; } \\
\boldsymbol{P}\left(\boldsymbol{X}_{5}\right)= & {[0.284413218566582,0.284413218566782], } \\
& {[-2.373999555338525,-2.373999555338325] ; } \\
\boldsymbol{P}\left(\boldsymbol{X}_{6}\right)= & {[0.260588744512268,0.260588744512469], } \\
& {[0.376338245290841,0.376338245291041] . }
\end{aligned}
$$

where $\boldsymbol{P}\left(\boldsymbol{X}_{i}\right)$ means interval extension $\boldsymbol{P}$ at $\boldsymbol{X}_{i}, i=3,4,5,6$. Obviously, $\boldsymbol{X}_{3}$, $\boldsymbol{X}_{4}$ and $\boldsymbol{X}_{5}$ should be deleted from $\mathrm{X}$.

Therefore, the isolated real root interval of $S A S$ is

$$
\begin{aligned}
\boldsymbol{X}_{6}= & {[0.560588744512268,0.560588744512469], } \\
& {[0.376338245290841,0.376338245291041] . }
\end{aligned}
$$

\subsection{Comparison experiments}

Algorithm 6 has been implemented in Matlab 2010a named real_roots_semi. In this subsection, we do some experiments (see Appendix A) to compare it with Maple package Discover described in [16] on the platform of Maple 15 classic work sheet. All experiments are carried out on a Dell PC with Intel Core i3-2120 at 3.30GHZ and 4GB of RAM in Windows 7 .

Table 1 gives the comparison of execution time. In the first row, $M_{-} d$, $N_{-} v$ and $N_{-} p$ denote the max degree, number of variables, number of real points of the semi-algebraic system. And $\infty$ means the program out of memory. Among these examples, A.5 is from [17], the other examples are chosen randomly. As shown in Table 1, Discover is faster than real_root_semi for Example A.1 and A.5, since these semi-algebraic system are simple. When the system becomes more complicated, real_root_semi is more efficient than Discover. From table 1 we can also find that the costs of example A.6, A.7 and A.9 are much higher than those of other examples compared because these examples can satisfy the inequations. 


\section{Applications}

Most engineering problems can be reduced to solving nonlinear equations. In this section, some applications from robot kinematic, piecewise algebraic curve, chemical engineering, circuit design are tested to show the efficiency of our algorithm.

\subsection{Polynomial system}

In this subsection, we first investigate applications of our algorithm to some polynomial systems.

Example 4.1. Production of synthesis gas in an adiabatic reactor 34].

$$
\left\{\begin{array}{l}
x_{1} x_{7}+2 x_{2} x_{7}+x_{3} x_{7}-2 x_{6}=0 . \\
x_{7}\left(x_{3}+x_{4}+2 x+5\right)-2=0 \\
7 x_{1}+7 x_{2}+7 x_{5}-1=0 . \\
x_{1}+x_{2}+x_{3}+x_{4}+x_{5}-1=0 . \\
400 x_{1} x_{4}^{3}-178370 x_{3} x_{5}=0 . \\
x_{1} x_{3}-2.6058 x_{2} x_{4}=0 . \\
-28837 x_{1} x_{7}-139009 x_{2} x_{7}-78213 x_{3} x_{7}+18927 x_{4} x_{7}+8427 x_{5} x_{7}+ \\
13492-10690 x_{6}=0 . \\
0 \leq x_{i} \leq 1, i=1,2,3,4,5 . \\
0 \leq x_{i} \leq 5, i=6,7 .
\end{array}\right.
$$

The above system of equations represents three atom balances, a mole fraction constraint, two equilibrium relations, and an energy balance equations. We obtain 8 real roots of this equation through 0.536665 seconds

\begin{tabular}{|c|c|c|c|c|c|}
\hline Example No. & Max degree & $N_{-} v$ & $N_{-} p$ & Discover & real_roots_semi \\
\hline A.1 & 2 & 3 & 1 & 0.062 & 0.259715 \\
\hline A.2 & 3 & 3 & 1 & 0.483 & 0.188181 \\
\hline A.3 & 4 & 3 & 0 & 219.759 & 0.461846 \\
\hline A.4 & 2 & 4 & 1 & 13.947 & 0.302547 \\
\hline A.5 & 3 & 4 & 1 & 0.31 & 0.756411 \\
\hline A.6 & 4 & 4 & 3 & 9.734 & 6.106957 \\
\hline A.7 & 2 & 4 & 2 & 13.821 & 1.942310 \\
\hline A.8 & 4 & 5 & 5 & $\infty$ & 0.794765 \\
\hline A.9 & 2 & 6 & 5 & $\infty$ & 11.272852 \\
\hline
\end{tabular}

Table 1: Time comparison, units:s. 
and 8 interval iterations for the 8 solution, then remove 7 real roots after 0.061837 seconds. Let the midpoint of interval be the approximate root of the problem, then

$$
\boldsymbol{x}=[0.322870839476541,0.009223543539188,0.046017090960632,
$$
0.618171675070824, 0.003716850952815, 0.576715395935549, 2.977863450791145].

Example 4.2. Robot kinematic problem [35].

$$
\left\{\begin{array}{l}
\left(0.004731 x_{3}-0.1238\right) x_{1}-\left(0.3578 x_{3}+0.001637\right) x_{2}+x_{7}-0.9338 x_{4} \\
-0.3571=0 . \\
0.2238 x_{1} x_{3}+0.7623 x_{2} x_{3}+0.2638 x_{1}-x_{7}-0.07745 x_{2}-0.6734 x_{4} \\
-0.6022=0 \\
x_{6} x_{8}+0.3578+0.004731 x_{2}=0 . \\
-0.7623 x_{1}+0.2238 x_{2}+0.3461=0 \\
x_{1}^{2}+x_{2}^{2}-1=0 . \\
x_{3}^{2}+x_{4}^{2}-1=0 . \\
x_{5}^{2}+x_{6}^{2}-1=0 . \\
x_{7}^{2}+x_{8}^{2}-1 \\
x_{i} \geq-1, x_{i} \leq 1, i=1,2, \cdots, 8
\end{array}\right.
$$

All 16 real solutions were founded in 0.826805 seconds of CPU time, which is more efficient than 3.783 seconds CPU time in [37] to solve the problem.

Example 4.3. This example addresses the equilibrium of the products of a hydrocarbon combustion process [36]. The problem is reformulated in the 'element variables' space.

$$
\left\{\begin{array}{l}
y_{1} y_{2}+y_{1}-3 y_{5}=0 \\
2 y_{1} y_{2}+y_{1}+3 R_{10} y_{2}^{2}+y_{2} y_{3}^{2}+R_{7} y_{2} y_{3}+R_{9} y_{2} y_{4}+R_{8} y_{2}-R y_{5}=0 \\
2 y_{2} y_{3}^{2}+R_{7} y_{2} y_{3}+2 R_{5} y_{3}^{2}+R_{6} y_{3}-8 y_{5}=0 \\
R_{9} y_{2} y_{4}+2 y_{4}^{2}-4 R y_{5}=0 \\
y_{1} y_{2}+y_{1}+R_{10} y_{2}^{2}+y_{2} y_{3}^{2}+R_{7} y_{2} y_{3}+R_{9} y_{2} y_{4}+R_{8} y_{2}+R_{5} y_{3}^{2} \\
+R_{6} y_{3}+y_{4}^{2}-1=0 \\
y_{i}-0.0001 \geq 0,100-y_{i} \geq 0, i=1,2, \cdots, 5
\end{array}\right.
$$

The value of parameter $R_{i}, i=1,2, \cdots, 10$ can be found in [36]. The method in [37] finds the single solution after 217.7 seconds of CPU time. Using our method, we only use 0.2964 seconds CPU time.

Example 4.4. This example for computing the real roots of piecewise algebraic curve is modified from [3]. 


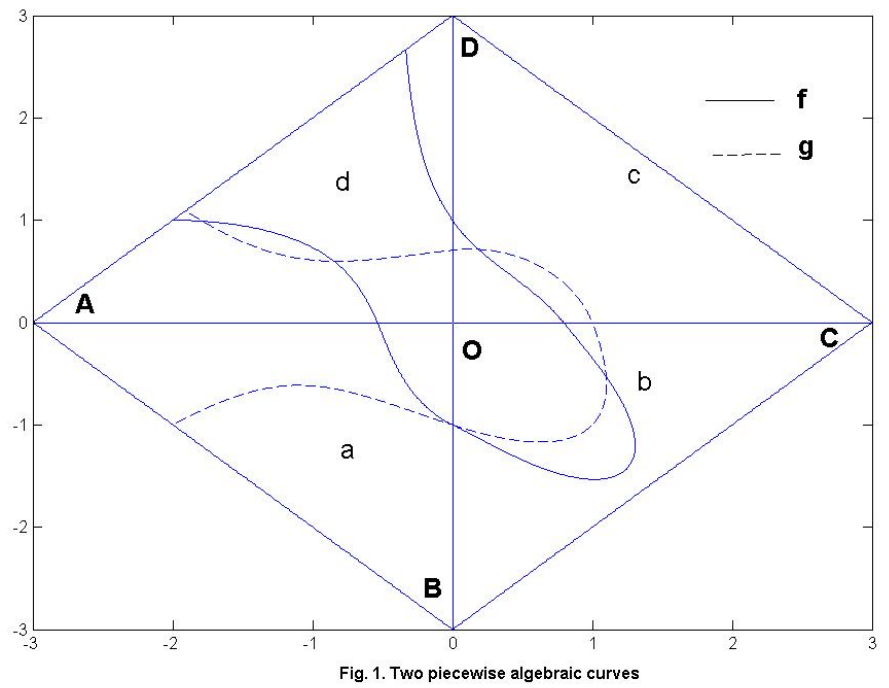

Let $\Delta=\{a, b, c, d\}$ be a regular triangulation of rectangular domain $A B C D$ in $\mathbb{R}^{2}$, where $a=[A O B], b=[B O C], c=[C O D]$ and $d=[A O D]$, where $A=(-3,0), B=(0,-3), C=(3,0) D=(0,3)$ and $O=(0,0), f$ and $g$ are algebraic curves (See Fig. 1).

Suppose that $f, g \in S_{3}^{1}(\Delta)$ and

on cell $a:\left\{\begin{array}{l}\left.f_{1}\right|_{a}=x^{3}-y^{3}+3 y^{2}+x y-3 x-4 . \\ \left.g_{1}\right|_{a}=x^{3}-y^{3}+2 y x+4 x^{2}-1\end{array}\right.$

on cell $b:\left\{\begin{array}{l}\left.f_{1}\right|_{b}=\left.f_{1}\right|_{a}+x^{2}(2 x+3 y+4) . \\ \left.g_{1}\right|_{b}=\left.g_{1}\right|_{a}+x^{2}(x+y-4)\end{array}\right.$

on cell $c:\left\{\begin{array}{l}\left.f_{1}\right|_{c}=\left.f_{1}\right|_{b}+y^{2}(x+y+5) . \\ \left.g_{1}\right|_{c}=\left.g_{1}\right|_{b}+y^{2}(2 x+y+1) .\end{array}\right.$

On cell $d:\left\{\begin{array}{l}\left.f_{1}\right|_{d}=\left.f_{1}\right|_{a}+y^{2}(x+y+5) . \\ \left.g_{1}\right|_{d}=\left.g_{1}\right|_{a}+y^{2}(2 x+y+1) .\end{array}\right.$

We take the cell $a$ for instance, isolating the intersection points of $\left\{\left.f_{1}\right|_{a},\left.g_{1}\right|_{a}\right\}$ 
lying in $a$ can be converted to isolating the real roots of the following semialgebraic system:

$$
\left\{\begin{array}{l}
x^{3}-y^{3}+3 y^{2}+x y-3 x-4=0 . \\
x^{3}-y^{3}+2 y x+4 x^{2}-1=0 \\
-x \geq 0,-y \geq 0, x+y+3 \geq 0
\end{array}\right.
$$

Using the algorithm real_root_semi, we know that there is only one real root lying in $a$. Similarly, we conclude that $\left\{\left.f_{1}\right|_{c},\left.g_{1}\right|_{c}\right\}$ has one real point in the interior of $c$, and respectively, $\left\{\left.f_{1}\right|_{b},\left.g_{1}\right|_{b}\right\}$ and $\left\{\left.f_{1}\right|_{d},\left.g_{1}\right|_{d}\right\}$ has two common points in the cell of $b$ and $d$.

Let the polynomial $\left\{\left.f_{1}\right|_{b},\left.g_{1}\right|_{b}\right\}$ be inequations, and append it to system (9), we conclude that $a$ and $b$ have a common real point. Hence, $f$ and $g$ have 5 real points. Methods given by Wang, et al., based on interval method, Groebner basis in [1, 2, 3, 4, 5] can also find the commom points of piecewise algebraic curve, but they did not consider these points which lie in the boundary.

\subsection{Transcendental functions system}

In practice, systems of transcendental functions appear in many applications [34, 35], e.g., logarithm functions, exponential functions and trigonometric functions.

As far as we know, there exists three kinds of numerical methods to solve nonlinear equations: homotopy continuation method, Newton's iteration and interval bisection method. Each kind of algorithm has its advantages and disadvantages. Homotopy continuation method is a global convergence algorithm, but it can only be used for solving polynomial equations. Given an appropriate initial point, the convergence of Newton's method is typical local quadratic, but how to choose the initial point is a difficult problem. The interval method is unacceptable in computation when some conditions are complicated, such as more variables, the huge width of the initial interval and the more accuracy of the final result.

The basic idea of our method is to replace the exponential function by its Taylor expansion first. In this step, rather than using the initial interval, we reduce the width of the initial interval using interval algorithm, until the errors between the exponential function and its Taylor expansion is smaller than a tolerance. We then apply homotopy method to the polynomial system to obtain the approximate real roots. At last, the real roots of transcendental 
functions are found based on these approximate real roots using the Newton's method.

In the following, we apply this idea to solve a circuit design problem from [38].

Example 4.5. Circuit design problem with extraordinary sensitivities to small perturbations.

$$
\left\{\begin{array}{l}
\left(1-x_{1} x_{2}\right) x_{3}\left(e^{\left(x_{5}\left(g_{1 k}-g_{3 k} x_{7} 10^{-3}-g_{5 k} x_{8} 10^{-3}\right)\right)}-1\right)-g_{5 k}+g_{4 k} x_{2}=0 . \\
\left(1-x_{1} x_{2}\right) x_{4}\left(e^{\left(x_{6}\left(g_{1 k}-g_{2 k}-g_{3 k} x_{7} 10^{-3}+g_{4 k} x_{9} 10^{-3}\right)\right)}-1\right)-g_{5 k} x_{1}+g_{4 k}=0 . \\
x_{1} x_{3}-x_{2} x_{4}=0 . \\
k=1,2,3,4 \\
x_{i} \in[0,10], i=1, \cdots, 9 .
\end{array}\right.
$$

The constant $g_{i k}$ are given by the following matrix

$$
\left(\begin{array}{cccc}
0.4850 & 0.7520 & 0.8690 & 0.9820 \\
0.3690 & 1.2540 & 0.7030 & 1.4550 \\
5.2095 & 10.0677 & 22.9274 & 20.2153 \\
23.3037 & 101.7790 & 111.4610 & 191.2670 \\
28.5132 & 111.8467 & 134.3884 & 211.4823
\end{array}\right)
$$

Step 1: Since the exponential function in system (10) only contains variables $x_{i}, i=5,6,7,8,9$, we only bisect the intervals of these variables by interval Newton iteration. This is different from other algorithms. After 10 times interval iterations for each variable, we have the last interval of these variables

$$
\begin{aligned}
& {[7.998046875,8.007812500],} \\
& {[7.998046875,8.007812500],} \\
& {[5.000000000,5.009765625],} \\
& {[0.996093750,1.005859375],} \\
& {[1.992187500,2.001953125] .}
\end{aligned}
$$

So we only need 50 bisections, and this progress takes about 9 seconds. Now, we compute the interval extension of $g_{1 k}=x_{5}\left(g_{1 k}-g_{3 k} x_{7} 10^{-3}-\right.$ $\left.g_{5 k} x_{8} 10^{-3}\right)$ and $g_{2 k}=x_{6}\left(g_{1 k}-g_{2 k}-g_{3 k} x_{7} 10^{-3}+g_{4 k} x_{9} 10^{-3}\right)$, and using the Taylor expansion of the exponential function in the midpoint of the interval extension of $g_{1 k}$ and $g_{2 k}$ to replace $e^{g_{1 k}}$ and $e^{g_{2 k}}$ for $k=1,2,3,4$ in equation 
(10). At this point, we use the second order Taylor expansion for $e^{g_{14}}$ and the first order for other exponential functions. Note that the errors generated by this step are not more than $10^{-2}$.

Step 2: For the polynomial system obtained from step 1, there exists 37 homotopy paths, but only 4 roots are founded using homotopy algorithm, and only 2 of them are real roots. This process takes 1.469615 seconds.

Step 3: Let the two real points be the initial point of the Newton's iteration for system (10). After 0.092 seconds we find that this two points converge to the same point.

$\hat{\boldsymbol{x}}=[.899999952618, .449987471886,1.00000648241,7.99997144063,5.00003127610$, $.999987723423,2.00006854190,7.99969268290,2.00005248366]$.

Compared with $\hat{\boldsymbol{x}}$, we find that the errors between $\hat{\boldsymbol{x}}$ and the initial point from step 2 is 1.2602 and 0.0017 , respectively. The last one is close to $\hat{\boldsymbol{x}}$ which ensure the convergence of the Newton iterative algorithm in this step.

From the above description, we totally use about 11 seconds to find the real point of equation (10), which is much less than 436.5 seconds in [37.

\section{Conclusion}

For a class of semi-algebraic system, this paper presents a numerical method for isolating the real roots. The algorithm first obtains the isolated intervals of a zero-dimensional polynomial system using hybrid technique [22], and substitute it into the inequations, nonnegative system, and positive systems to remove some intervals. Such implementation will be efficient if the interval extension of constrained equation does not contain zero at the isolated real root interval of the system. Otherwise, we give a complete numerical algorithm to determine if a polynomial equation is equal to zero. We implement our algorithm in Matlab environment. Many random examples have been checked along with comparison to famous software Discover . At last, this new method is used to solve problems originated from several applications. For transcendental functions, we give an idea for solving this problem which is a combination of Newton's method, homotopy algorithm

and interval arithmetic. However, the number of bisections, the order of Taylor expansion and the error control are deserved to further study.

\section{Acknowledge}

The authors especially thank associate professor Jinming Wu for the help of discussion about the piecewise algebraic curve. 


\section{References}

[1] Renhong Wang, Xiaolei Zhang, Interval iterative algorithm for computing the piecewise algebraic variety, Journal of Computers and Mathematics with Applications. 56 (2008) 565-571.

[2] Xiaolei Zhang, Renhong Wang, Isolating the real roots of the piecewise algebraic variety, Journal of Computers and Mathematics with Applications. 57 (2009) 565-570.

[3] Jinming $\mathrm{Wu}$, Real intersection points of piecewise algebraic curves, Applied Mathematics Letters. 25 (2012) 1299-1303.

[4] F. G. Lang, R. H. Wang, Intersection points algorithm for piecewise algebraic curves based on Groebner bases Journal of Applied Mathematics and Computing. 29 (2009) 357-366.

[5] R. H. Wang, J. M. Wu, Real roots isolation of spline functions, Journal of Applied Mathematics and Computing. 26 (2008) 69-75.

[6] D. Angeli, J. E. Ferrell, Jr. and E. D. Sontag, Detection of multistablity, bifurcations, and hysterssis in a large class of biological positive-feedback systems, In Proc. Nat.Acsad.Sci.USA 1822-1827, 2004.

[7] D. Wang and B. Xia. Stability analysis of biological systems with real solutions classification. In Proc. ISSAC 2005, 354-361, ACM Press, New York, (2005).

[8] Chen Yinghua, Xia Bican, Yang Lu, Zhan Naijun, Zhou Chao chen, Discovering Non-linear Ranking Functions by Solving Semi-algebraic Systems, In Theoretical Aspects of Computing ICTAC 2007, volume 4711 of LNCS, 34-49. Springer, 2007.

[9] Bican Xia, Lu Yang, Naijun Zhan. Program Verification by Reduction to Semi-Algebraic Systems Solving. In Proc. Leveraging Applications of Formal Methods, Verification and Validation, 277-291, 2009.

[10] Yang, L., Hou, X. R., Xia, B. C, A complete algorithm for automated discoving of a class of inequality-type theorem. Sci. China Ser.F, 44 (2001) 33-49. 
[11] G. E. Collins, R. Loos, Real zeros of polynomials. in: Buchberger, B. and Collins, G.E. and Loos, R. eds., Computer Algebra: Symbolic and Algebraic Computation, Springer-Verlag, 1982, pp 83-94.

[12] A. G. Akritas, A. V. Bocharov, A. W. Strz'ebonski. Implementation of real root isolation algorithms in Mathematica. in: Abstracts of the International Conference on Interval and Computer-Algebraic Methods in Science and Engineering (Interval94), St. Petersburg, Russia, March 7-10, pp. 23-27, 1994.

[13] A. G. Akritas, A. W. Strz'ebonski, A Comparative Study of Two Real Root Isolation Methods, Nonlinear Analysis: Modelling and Control. 10 (2005) 297-304.

[14] Ting Zhang and Bican Xia. A New Method for Real Root Isolation of Univariate Polynomials, Mathematics in Computer Science. 1 (2007) 305-320.

[15] Bernard Mourrain, Fabrice Rouillier, Marie-Franfcoise Roy, Bernstein's basis and real root isolation, Rapport de recherche 5149, INRIARocquencourt (2004).

[16] B.C.Xia and L. Yang, An algorithm for isolating the real solutions of semi-algebra systems, Journal of Symbolic Computation. 34 (2002) 461477 .

[17] Bican Xia, Ting Zhang, Real soltion iaolation using interval arithmetic, Journal of Computers And Mathematics with Applications. 52 (2006) 853-860.

[18] F. Boulier, Chen C.B., F. Lemaire, and Marc Moreno Maza. Real Root Isolation of Regular Chains. In Proc. 2009 Asian Symposium on Computer Mathematics (ASCM 2009), 1-15, 2009.

[19] Cheng J.S, Gao X.S and Guo L.L. Root isolation of zero-dimensional polynomial systems with linear univariate representation. Journal of Symbolic Computation, 47 (2012) 843-858.

[20] Cheng J.S., Gao X.S. and Yap C.K, Complete Numerical Isolation of Real Zeros in General Triangular Systems. In Proc. 2007 Internat. Symp. Symbolic Algebraic Comput, 92-99, 2007. 
[21] F, Rouillier, Solving Zero-Dimensional Systems Through the Rational Univariate Representation, Applicable Algebra in Engineering, Communication and Computing, 9 (1999) 433-461.

[22] Shen F. The Real Roots Isolation of Polynomial System Based on Hybrid Computation. Master degree thesis, Peking University, April, 2012.

[23] R. E. Moore. IntervalAnalysis. Prentice-Hall. Englewood Cliffs,1966.

[24] D. R. Wang,et al., Interval Methods for Nonlinear Equations, Shanghai Scientific and Technical Publishers, 1987.

[25] R. E. Moore, A test for existence fo solutions to nonlinear systems, SIAM J.Numer.Anal. 14 (1977) 611-615.

[26] Tien-Yien Li. Solving Polynomial Systems By Polyhedral Homotopies. Taiwanese Journal of Mathematics. 3 (1999) 251-279.

[27] Alexander P. Morgan, A homotopy for solving polynomial systems, Applied Mathematics and Computation. 18 (1986) 173-177.

[28] J. Verschelde, P. Verlinde, R. Cools, Homotopies exploiting Newton polytopes for solving sparse polynomial system, SIAM J. Numer. Anal. 31 (1994) 915-930.

[29] Li T.Y. Numercial solution of polynomial systems by homotopy continuation methods, Handbook of Numerical Analysis. 11 (2003) 209-304.

[30] A. Sommese, C. Wampler, The numerical solutions of system of polynomials: Arising in Engineering and Science. World Scientific, 2005.

[31] Li T. Y., HOM4PS-2.0. http://hom4ps.math.msu.edu/HOM4PS soft.htm, 2008.

[32] J. Verschelde. PHCpack. http://homepages.math.uic.edu/jan/PHCpack /phcpack.html, 1999.

[33] C. Wampler. HomLab. http://nd.edu/cwample1/HomLab/main.html, 2005.

[34] M. Kuno, J. D. Seader, Computing all real solutions to systems of nonlinear equations with global fixed-point homotopy, Ind. Eng. Chem. Res. 27 (1988) 1320-1329. 
[35] C. D. Maranas and C. A. Floudas, Finding all solutions of nonlinearly constrained systems of equations, Journal of Global Optimization. 7 (1995) 143-182.

[36] K. Meintjes and A. P. Morgan. Chemical Equilibrium Systems as Numerical Test Problmes, ACM Transactions on Mathematical Software. 16 (1990) 143-151.

[37] M. D. Stuber, V. Kumar, P. I. Barton. Nonsmooth exclusion test for finding all solutions of nonlinear equations, BIT Numer Math . 50 (2010) 885-917.

[38] H. Ratschek and J. Rokne. Experiments using interval analysis for solving a circuit design problem, Journal of Global Optimization. 3 (1993) 501-518.

\section{Appendix A.}

$$
\begin{aligned}
& \left\{\begin{array}{l}
-3+95 x_{3}+29 x_{1} x_{2}+63 x_{1} x_{3}+37 x_{2}^{2}-36 x_{3}^{2}=0 . \\
-2 x_{1}+46 x_{2}+4 x_{1} x_{2}+50 x_{1} x_{3}+61 x_{3} x_{2}-99 x_{3}^{2}=0 . \\
72-49 x_{1}+13 x_{2}-15 x_{1}^{2}-12 x_{2}^{2}+23 x_{3}^{2}=0 . \\
3 x_{2}+2 x_{1}-1 \geq 0,3 x_{1}-4 x_{2}-3>0
\end{array}\right. \\
& \left\{\begin{array}{l}
-85 x_{1}-76 x_{2}+75 x_{3}-41 x_{2}^{2}-84 x_{2}^{3}=0 . \\
-39+26 x_{1} x_{3}+47 x_{1}^{3}-47 x_{1}^{2} x_{2}+91 x_{2}^{2} x_{1}+43 x_{3} x_{1} x_{2}=0 . \\
16 x_{1}+25 x_{3}+13 x_{1} x_{2}+x_{2}^{2}-8 x_{3} x_{1}^{2}-74 x_{2} x_{3}^{2}=0 \\
19 x_{3}+10 x_{1} x_{3}+53 x_{3} x_{2}-97 x_{3} x_{1}^{2}+57 x_{2}^{2} x_{1}+68 x_{2} x_{3}^{2} \geq 0 \\
47 x_{1} x_{2}+58 x_{3} x_{2}+30 x_{3}^{2}+55 x_{3} x_{1}^{2}+56 x_{3} x_{1} x_{2}+55 x_{3}^{3} \geq 0 \\
48 x_{2}+34 x_{1}^{2}+32 x_{1}^{3}-41 x_{2}^{2} x_{1}-56 x_{2}^{3}+63 x_{2} x_{3}^{2} \geq 0 \\
27+11 x_{1}+46 x_{3}-42 x_{1} x_{3}-60 x_{1}^{3}-48 x_{2}^{2} x_{3} \geq 0
\end{array}\right. \\
& \left\{\begin{array}{l}
-49 x_{2}+31 x_{3} x_{2}+73 x_{1} x_{3}^{2}+95 x_{2}^{2} x_{1}^{2}+68 x_{1} x_{2} x_{3}^{2}-29 x_{1} x_{3}^{3}=0 . \\
37+5 x_{1}^{2}-36 x_{3}^{2}-57 x_{2} x_{3}^{2}+85 x_{1} x_{3}^{3}+80 x_{2}^{2} x_{3}^{2}=0 . \\
30 x_{2}^{2}-3 x_{3} x_{2}-56 x_{1}^{2} x_{2}-91 x_{2}^{2} x_{1}^{2}-70 x_{3} x_{2}^{2} x_{1}+42 x_{2}^{4}=0 \\
62+96 x_{2}-51 x_{3}+89 x_{1}^{2}+14 x_{2}^{2} x_{1}-79 x_{2}^{2} x_{3}>0 \\
86+57 x_{1}-35 x_{1}^{2}+57 x_{2}^{2}+28 x_{3}^{2}+63 x_{3} x_{1}^{2}>0 \\
-61 x_{1}+45 x_{3}-50 x_{2}^{2}-22 x_{1}^{3}+48 x_{1}^{2} x_{2}-82 x_{2} x_{3}^{2}>0 \\
-85 x_{3} x_{2}-44 x_{3} x_{1}^{2}-31 x_{2}^{2} x_{1}+45 x_{1} x_{3} x_{2}+49 x_{2}^{3}-58 x_{3}^{3} \geq 0
\end{array}\right.
\end{aligned}
$$




$$
\begin{aligned}
& \left\{\begin{array}{l}
94-99 x_{1} x_{3}+46 x_{2} x_{4}+41 x_{3}^{2}+95 x_{3} x_{4}=0 . \\
-59 x_{1}-15 x_{4}+30 x_{1} x_{2}-57 x_{1} x_{3}+50 x_{2}^{2}+62 x_{2} x_{3}=0 . \\
53-9 x_{1}-99 x_{4}+12 x_{1} x_{4}+20 x_{2} x_{4}+99 x_{4}^{2}=0 \\
-82-22 x_{3}-60 x_{2}^{2}+3 x_{2} x_{4}-83 x_{3} x_{4}-84 x_{4}^{2}=0 \\
-74 x_{2} x_{3}+87 x_{3}^{2}+68 x_{4} x_{1}^{2}-88 x_{3} x_{2}^{2}-88 x_{2} x_{3}^{2}-87 x_{2} x_{3} x_{4} \geq 0
\end{array}\right. \\
& \left\{\begin{array}{l}
2-6.5 x_{1}+x_{1}^{2} x_{2}-0.5 x_{3}=0 . \\
6 x_{1}-x_{1}^{2} x_{2}-5 x_{4}+5 x_{2}=0 . \\
2-6.5 x_{3}+x_{3}^{2} x_{4}-0.5 x_{1}=0 \\
6 x_{3}-x_{3}^{2} x_{4}+1+0.5 x_{2}-0.5 x_{4}=0 \\
x_{1}>0, x_{2}>0, x_{3}>0, x_{4}>0
\end{array}\right. \\
& \left\{\begin{array}{l}
9 x_{1}^{2}-20+x_{1}^{2} x_{4}-5 x_{4}-x_{1}^{4}-4 x_{1}^{3} x_{2}+20 x_{1} x_{2}-4 x_{1}^{2} x_{2} x_{4}+20 x_{2} x_{4} \\
+3 x_{1}^{2} x_{3}^{2}-15 x_{3}^{2}=0 . \\
3 x_{1}^{2} x_{2}^{2}-39 x_{1}^{2}+4 x_{1} x_{2}^{3}-52 x_{1} x_{2}-5 x_{3} x_{1} x_{2}^{2}+65 x_{1} x_{3}+3 x_{1} x_{2}^{2} x_{4} \\
-39 x_{1} x_{4}-3 x_{2}^{4}+39 x_{2}^{2}=0 . \\
-7-7 x_{2}+4 x_{1} x_{3}-2 x_{1} x_{4}+5 x_{2} x_{3}+5 x_{2} x_{4}=0 . \\
3+2 x_{1}+3 x_{3}-3 x_{1}^{2}+5 x_{1} x_{2}-x_{3} x_{4}=0 . \\
x_{1}^{2}-5 \neq 0, x_{2}^{2}-13 \neq 0 .
\end{array}\right.
\end{aligned}
$$

$$
\left\{\begin{array}{l}
2 x_{1}^{3}-3 x_{1}^{2} x_{2}-8 x_{1}^{2}-5 x_{1} x_{2}^{2}-12 x_{1} x_{2}+5 x_{1}^{2} x_{3}-12 x_{1} x_{3} x_{2}-20 x_{1} x_{3} \\
+4 x_{1}^{2} x_{4}-12 x_{1} x_{2} x_{4}-16 x_{1} x_{4}-12 x_{2}^{3}-16 x_{2}^{2}-9 x_{2}^{2} x_{3}-12 x_{2} x_{3}=0 . \\
50 x_{3}-65-20 x_{1} x_{3}+26 x_{1}-30 x_{1} x_{3} x_{2}+39 x_{1} x_{2}-70 x_{2} x_{3}^{2}+91 x_{2} x_{3} \\
-70 x_{3}^{3}+91 x_{3}^{2}-40 x_{3}^{2} x_{4}+52 x_{3} x_{4}=0 . \\
5 x_{1}-2 x_{3}-2 x_{1} x_{4}-2 x_{2} x_{4}-x_{3}^{2}-7 x_{4}^{2}=0 . \\
-7-7 x_{1}+2 x_{1} x_{2}-7 x_{2} x_{4}-3 x_{3} x_{4}+5 x_{4}^{2}=0 . \\
x_{1}-3 x_{2}-4 \neq 0, x_{3}-1.3 \neq 0 .
\end{array}\right.
$$




$$
\left\{\begin{array}{l}
66 x_{4} x_{2}^{3} x_{1}-44 x_{1}^{2} x_{2} x_{4}+66 x_{1} x_{2} x_{4}-165 x_{4} x_{5}^{2} x_{2}^{2}+110 x_{4} x_{5}^{2} x_{1}-165 x_{4} x_{5}^{2}=0 \\
-10 x_{1}+62 x_{3}-82 x_{4}+80 x_{5}-44 x_{2}^{2}+71 x_{3} x_{4}=0 . \\
74 x_{1} x_{2}+72 x_{1} x_{4}+37 x_{2} x_{4}-23 x_{2} x_{5}+87 x_{3} x_{5}+44 x_{4}^{2}=0 \\
11-49 x_{2}-47 x_{5}+40 x_{1}^{2}-81 x_{2} x_{3}+91 x_{2} x_{4}=0 \\
-28 x_{1}+16 x_{2}+30 x_{4}-27 x_{1} x_{2}-15 x_{1} x_{4}-59 x_{3} x_{4}=0 \\
3 x_{2}^{2}-2 x_{1}+3 \neq 0, x_{4} \neq 0
\end{array}\right.
$$

$$
\left\{\begin{array}{l}
36 x_{2}+91 x_{3}-22 x_{2} x_{3}+51 x_{3} x_{6}-27 x_{4} x_{5}+50 x_{5}^{2}=0 . \\
25 x_{1}+31 x_{2}-27 x_{4}+65 x_{1} x_{2}+88 x_{2} x_{5}+10 x_{3} x_{5}=0 . \\
95 x_{2}+68 x_{1} x_{2}-29 x_{1} x_{6}+5 x_{2} x_{4}-26 x_{3}^{2}-51 x_{3} x_{4}=0 . \\
5-36 x_{4}-57 x_{1} x_{2}+85 x_{3}^{2}+80 x_{4} x_{6}+90 x_{6}^{2}=0 . \\
65 x_{1} x_{2}-12 x_{1} x_{5}+78 x_{1} x_{6}+5 x_{2}^{2}-63 x_{2} x_{6}-5 x_{3} x_{5}=0 . \\
-70+42 x_{1}+9 x_{1}^{2}-21 x_{1} x_{2}-27 x_{1} x_{5}-79 x_{6}^{2}=0 . \\
x_{4}^{2}+x_{5} x_{6}-2 \geq 0 . \\
3 x_{1}-4 x_{2}+4>0 .
\end{array}\right.
$$

